\title{
BreastCare
}

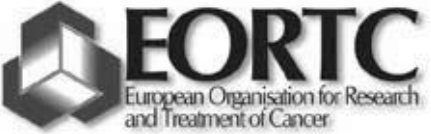

Gaston Demonty MD, Clinical Research Fellow EORTC Breast Cancer Group

Patrick Therasse MD, Coordinating Physician EORTC Breast Cancer Group - Director EORTC Data Center European Organisation for Research and Treatment of Cancer

EORTC Breast Cancer Group

www.eortc.be

\section{The EORTC Breast Cancer Group (EBCG)}

The aims of the European Organisation for Research and Treatment of Cancer (EORTC) are to develop, conduct, coordinate, and stimulate laboratory and clinical research in Europe to improve the management of cancer and related problems by increasing survival but also patients' quality of life. The EORTC Breast Cancer Group (EBCG) is a multidisciplinary group of oncologists, surgeons and radiotherapists working in breast cancer care in European hospitals. The main goal of the EBCG is to carry high quality international clinical trials covering all areas of breast cancer care, from in situ carcinoma to metastatic disease, investigating new anticancer agents in phase I/II trials and therapeutic questions of strategic importance in large phase III trials.

The EBCG is very active relating patient accrual. It recruited in the last 3 years an average of 877 patients/year in 48 medical centres across all its studies. These patients are included not only in EORTC led studies, but also in intergroup trials where the leading group (the one which designs and conducts the trial) is not the EORTC. EORTC is one of the founding organisations of the Breast International Group (BIG), a worldwide network of breast cancer research groups, since intergroup collaboration is essential, by avoiding duplication of efforts and waste of resources.

\section{EORTC Trials}

In the last years the EORTC started to become increasingly more active in the field of translational research.
As tools such as the microarray technology are becoming more important in current research, the EBCG incorporated translational research elements in their protocols. Translational research evaluating correlations between clinical outcomes and biologic tumour characteristics has become a high priority. The ongoing p53 trial (EORTC trial 10994) is a good example of this strategy. This study assesses the potential predictive value of p53 gene mutations in patients with locally advanced/inflammatory or large operable breast cancer prospectively randomized to a taxane versus a non-taxane regimen. Patients are treated in the neoadjuvant setting, which allows assessing clinical and pathological response after chemotherapy.

Another ambitious project of the EBCG is the MINDACT trial (EORTC trial 10041). This is an EORTC coordinated study conducted under the BIG/TRANSBIG network. It will use microarray technology to classify node negative early breast cancer patients into groups with high and low risk of distant relapse and compare this assessment to what is currently used in clinical practice, the latter taking into consideration traditional clinico-pathological factors. MINDACT aims to prospectively validate a prognostic signature, a genomic test developed with microarray technology that holds the promise to better define patient prognosis and therefore to better select those who need adjuvant chemotherapy. It will test the hypothesis that the use of this genetic signature will spare $10-20 \%$ of women who would normally receive adjuvant chemotherapy based on their clinicopathological factors sparing them from the inconvenience and morbidity of this therapy, without having any negative impact in overall survival.

\section{Medical Education}

The EORTC is deeply involved with medical education. The EBCG has an active fellowship programme, where young physicians are trained and work on group projects either at the EORTC Data Center, where they participate in setting up and conducting clinical trials, or at active institutions, where they conduct translational research projects. In addition, the EBCG organises every 2 years the European Breast Cancer Conference, jointly with EUSOMA and Europa Donna. This meeting is one of the most important breast cancer meetings in the world, and in its 2006 edition in Nice it attracted more than 5000 participants from around the globe. 